\title{
Instrumentation avancée en écoulement diphasique : rêve ou réalité ?
}

\author{
Advanced instrumentation for two-phase flow : dream or reality?
}

\author{
par H. Lemonnier \\ Commissariat à l'Energie Atomique, Grenoble \\ DTP/SMTH/LATA
}

The design and optimisation of industrial equipment could be made easier if validated computer multiphase flow software would exist. Two-phase flow model development is indeed stalled due to the lack of appropriate measuring techniques. Among the most necessary ones there are the local instantaneous diagnostic tools, obviously because computer software is based on average equations which require appropriate closure. The validation of these closure relations requires at least the experimental knowledge of the values of the local phase velocity, the interfacial area and phase fractions as well as some estimate of the velocity fluctuations. This paper reports recent progress in this field which have been presented at the last OECD-sponsored meeting on two-phase flow instrumentation held in March 1997 at Santa Barbara, CA (USA).

\section{I $\square$ INTRODUCTION}

Les besoins en techniques de mesure locales pour les écoulements diphasiques se répartissent approximativement selon trois catégories principales.

- La caractérisation globale où les informations sur la structure de l'écoulement comme la distribution des phases et la stationnarité sont les plus recherchées.

- La caractérisation quantitative où l'on veut déterminer une grandeur caractéristique du procédé ou du dispositif étudié pour en évaluer l'efficacité.

- Les techniques de référence où, au prix de l'adaptation des conditions d'écoulement, on veut déterminer avec la meilleure précision possible une grandeur caractéristique de l'écoulement.

Parmi la première catégorie, on pourra regrouper les techniques de visualisation et d'imagerie, comme l'imagerie d'impédance qui exploite la sensibilité de la réponse électrique d'un milieu à la distribution des phases. C'est selon le cas la constante diélectrique ou la conductivité dont on recherche la distribution à l'intérieur d'un domaine donné [1]. L'une comme l'autre de ces propriétés dépendent généralement de la fraction volumique de phase ou de la fonction de présence de phase. Des travaux récents [2,3] montrent que malgré des efforts importants, la résolution spatiale de cette technique est limitée et qu'elle n'est généralement mise en œuvre que dans des configurations exactement bidimensionnelles. Lemonnier et Peytraud [4] ont noté, après d'autres, que le problème de la reconstruction associé à la tomographie d'impédance était mal conditionné. En conséquence, ces auteurs montrent que les précisions de mesure nécessaires à une reconstruction de qualité sont hors d'atteinte technologique et que des images fantômes apparaissent toujours lorsque la configuration d'écoulement est vraiment tridimensionnelle.

Les techniques d'absorption photoniques ou neutroniques sont en revanche bien plus précises. En effet, le rayonnement incident n'est pas déformé par la composition du milieu traversé et, lorsque le rayonnement diffusé est éliminé par une collimation soignée, l'atténuation selon une direction ne dépend que des valeurs de la masse volumique le long d'un rayon géométrique. La cinéradiographie $\mathrm{X}$ ou neutronique donne ainsi d'excellentes images de milieux complexes comme ceux résultant de l'interaction d'un métal fondu avec de l'eau froide $[5,6,7]$. Ce type de technique fournit à la fois une information sur la structure de l'écoulement et une information quantitative sur les fractions volumiques moyennées sur le trajet du faisceau dans le milieu. Pour obtenir des données locales, il faut recourir aux procédures tomographiques où la distribution de masse volumique du milieu est déduite des projections selon toutes les directions [8]. Pour accéder, de plus, à la structure dynamique de l'écoulement, encore faut-il acquérir toutes les données simultanément. Une très belle solution technologique développée par Mitsubishi sera présentée plus loin [9]. Cette dernière technique, analogue à la tomodensitométrie médicale, se place résolument dans la catégorie des techniques de référence.

Les modèles d'écoulements diphasiques de nouvelle génération incorporent une équation d'évolution de l'aire interfaciale. En effet, il est apparu indispensable, pour modéliser correctement les interactions entre phases, de décrire précisément l'aire à travers laquelle ces transferts s'opèrent. De plus, dans certains équipements du génie chimique, comme 


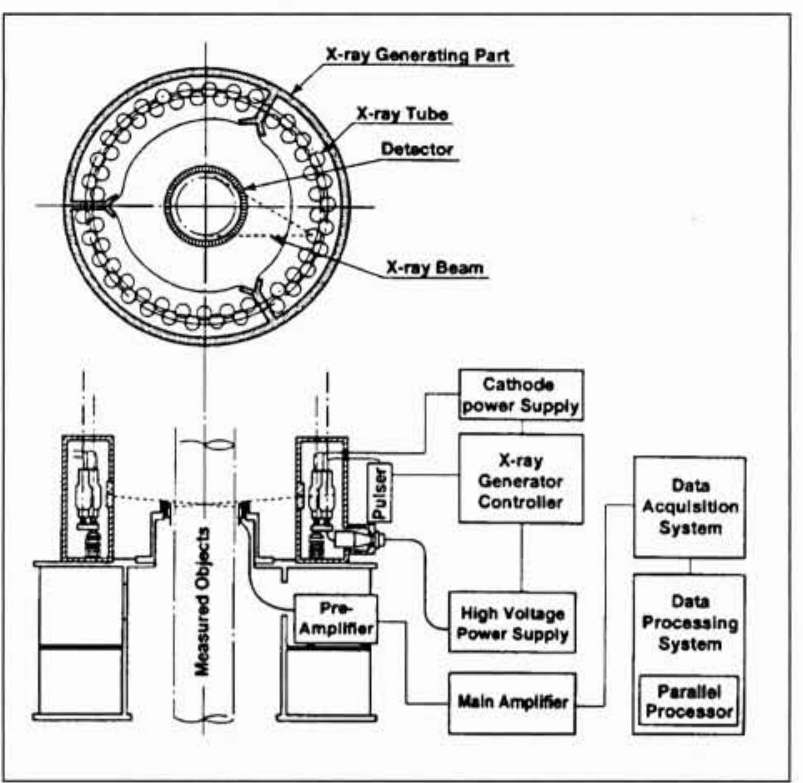

1. Schéma de la machine tomographique de Mitsubishi. D'après [9].

dans les réacteurs gaz-liquide, c'est une grandeur caractéristique du procédé. Il n'existe pas pour cette grandeur de méthode de mesure de référence en dehors de la fastidieuse méthode photographique. La méthode des sondes locales doubles, électriques [10] ou optiques [11] permet, en principe, de la mesurer localement et indépendamment du régime d'écoulement. Notons cependant qu'en écoulement à bulles, on peut déterminer l'aire interfaciale instantanée moyenne sur un trajet $[12,13]$ par des méthodes ultrasonores non intrusives.

Finalement, la grandeur la plus délicate à déterminer est sans conteste la vitesse moyenne de phase. On sait la déterminer par anémométrie thermique en écoulement à bulles $[10,14]$. Mais l'algorithme de traitement des signaux influe très notablement sur l'intensité des fluctuations mesurées [15]. Ces techniques sont d'usage limité aux écoulements possédant une direction privilégiée et à faible taux de turbulence et certains auteurs utilisent des techniques d'imagerie médicale basées sur la résonance magnétique nucléaire pour déterminer le champ le vitesse [16]. La quantification simultanée des vitesses moyennes de phase, et du taux de présence n'a été mise en œuvre, pour l'instant, qu'en conduite [17]. Il n'y a cependant pas d'objection de principe à l'obtention de données locales relatives à une section.

Les trois sections qui suivent font un point sur les derniers progrès en matière de détermination de grandeurs relatives aux écoulements diphasiques comme le taux de présence de phase, l'aire interfaciale locale ou volumique et la vitesse moyenne locale.

\section{II — LA TOMOGRAPHIE À RAYONS X}

Le principe de la tomographie à rayons $\mathrm{X}$ est mis en œuvre dans les tomodensitomètres médicaux. Il peut être transposé directement à un milieu diphasique pourvu que l'on puisse mesurer précisément la fraction linéique moyenne de phase selon une corde définie par :

$$
\overline{R_{G 1}} \triangleq \overline{L_{G}(t)} / L
$$

où $L_{\sigma}$ est la longueur de la corde traversant la phase $\mathrm{G}, \mathrm{L}$ est la longueur traversée et la barre représente la moyenne temporelle. Cette grandeur peut être déterminée par un équipement comprenant une seule source à laquelle fait face une barrette de détecteurs. Les différentes projections de l'objet sont obtenues en faisant tourner l'ensemble composé de la source et des détecteurs autour du milieu. Il est impossible, cependant, d'accéder à la structure instantanée de l'écoulement avec cette technologie étant donné que le temps de rotation de l'ensemble mécanique portant la source et les détecteurs est toujours très grand devant les temps caractéristiques d'évolution de l'écoulement.

Hori et ses collaborateurs ont mis au point une machine composée de 60 sources et 584 détecteurs fixes. Elle est schématiquement représentée à la figure 1. Chaque source émet un faisceau en éventail pendant un flash de $10 \mu \mathrm{s}$ et le cycle complet d'illumination par une source et de lecture des détecteurs lui faisant face dure $16,1 \mu \mathrm{s}$. De plus, les deux sources diamétralement opposées sont déclenchées simultanément. Une image est donc constituée de 30 cycles exécutés en séquence et peut donc être répétée avec une période de $0,5 \mathrm{~ms}$. La machine de Mitsubishi est donc une véritable caméra tomographique à 2000 images par seconde.

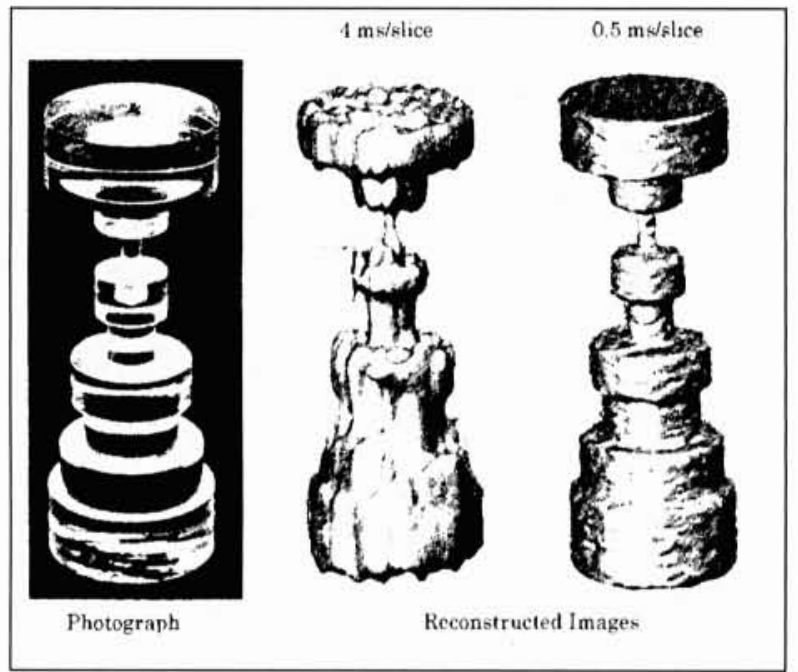

2. Photographie du fantôme (à gauche). Au centre, reconstruction avec 17 coupes espacées de $4 \mathrm{~ms}$. A droite, reconstruction avec 133 coupes espacées de 0,5 ms. D'après [9].

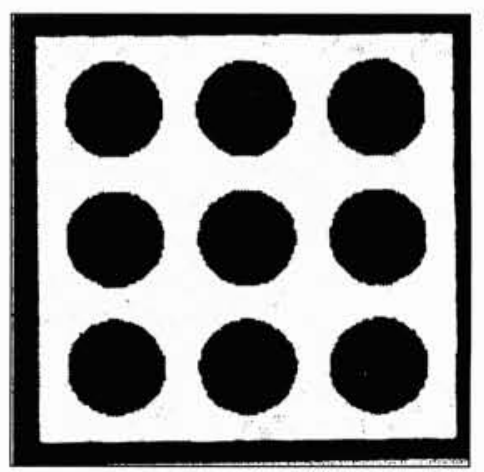

3. Reconstitution de l'écoulement dans un faisceau de tubes. En noir le boîtier et les tubes, en gris l'eau, en blanc, l'air. D'après [9]. 
La figure 2 illustre la qualité de la reconstruction et la finesse de la résolution. La maquette présentée à la figure 2 a une longueur de $100 \mathrm{~mm}$ et un diamètre de $35 \mathrm{~mm}$. Elle traverse le tomographe à une vitesse de $1,5 \mathrm{~m} / \mathrm{s}$. Avec une capture toutes les $4 \mathrm{~ms}$, on obtient une restitution acceptable. Avec une capture aux limites des possibilités de l'instrument, toutes les $0,5 \mathrm{~ms}$, on obtient une description parfaite du fantôme avec 133 coupes. L'incertitude relative sur le positionnement des fronts (sauts de masse volumique) est d'après Hori de $\pm 3 \%$ environ. Cette quantité correspond pour un objet de $35 \mathrm{~mm}$ à une incertitude absolue de $\pm 1 \mathrm{~mm}$. C'est une valeur proche de la résolution de la reconstruction que l'on peut calculer à partir du nombre de données indépendantes et du diamètre du volume de mesure, soit $1,6 \mathrm{~mm}$. La résolution verticale, c'est-à-dire l'épaisseur de tranche d'écoulement effectivement prise en compte est donnée par la hauteur physique du détecteur qui est de $1,8 \mathrm{~mm}$.

La figure 3 montre la reconstitution de l'écoulement dans un faisceau de $3 \times 3$ tubes d'aluminium placés au centre d'un boîtier en résine, en écoulement d'eau et d'air. Ce faisceau est analogue à celui d'un cœur de réacteur nucléaire (pas : $12,6 \mathrm{~mm}$ et diamètre : $9,5 \mathrm{~mm}$ ). L'assemblage comprend également 3 grilles de maintien espacées de $450 \mathrm{~mm}$. L'exemple fourni à la figure 3 représente une image extraite d'une série continue formant un véritable film à 2000 images par seconde. Les vitesses d'écoulement sont de $0,5 \mathrm{~m} / \mathrm{s}$ pour le gaz et le liquide, à la pression atmosphérique. Dans ces conditions, l'écoulement est plutôt agité et on observe le passage de poches de gaz entourant plusieurs tubes simultanément. C'est bien cette situation qui est décrite à la figure 3 . La fréquence des acquisitions permet des analyses fréquentielle et statistique valides. Les auteurs peuvent, en particulier, calculer le taux de présence instantané par sous-canal (espace compris entre 4 barreaux) et caractériser précisément l'influence de la géométrie de grilles de mélange sur la distribution des phases.

La fréquence élevée d'acquisition permet naturellement d'accéder au contenu fréquentiel du taux de vide moyen tout en conservant l'information sur la cohérence spatiale des structures. Ainsi, cette méthode possède également un potentiel important pour l'analyse des interactions fluide-structure en écoulement diphasique. De façon plus modeste, la tomographie à rayons $\mathrm{X}$ est, à notre connaissance, la seule méthode qui, par adaptation de techniques existantes, permet de déterminer le taux de présence moyen local, sans intrusion, pour un écoulement diphasique arbitraire et tridimensionnel. C'est donc la technique à mettre en auvre en priorité pour caractériser ces écoulements.

\section{III $\square$ LA DÉTERMINATION DES AIRES INTERFACIALES}

Les modèles d'écoulement diphasiques à deux fluides mono- ou multidimensionnels comportent de plus en plus souvent une équation de transport d'aire interfaciale. Cette dernière grandeur est directement liée à la modélisation des transferts interfaciaux et une équation de transport permet de rendre compte de l'influence sur l'aire interfaciale de différents mécanismes de relaxation ainsi que des phénomènes de coalescence et de fractionnement. La validation d'une telle équation repose sur la possibilité de déterminer expérimentalement cette grandeur.
Le principe de détermination de l'aire interfaciale repose sur la possibilité de mesurer le plus localement possible la fonction indicatrice de phase $\mathrm{X}_{\mathrm{k}}$

$$
X_{k}(x, t)= \begin{cases}I & \mathrm{x} \in \text { à la phase } \mathrm{k} \\ 0 & \mathrm{x} \notin \text { à la phase } \mathrm{k}\end{cases}
$$

Deux méthodes principales existent pour obtenir cette information : les sondes résistives, utilisées par exemple par l'équipe d'Ishii à Purdue University [10] ou les sondes optiques comme au CEA/Grenoble [11]. L'obtention de la fonction indicatrice de phase à partir d'un signal brut peut nécessiter un étalonnage, ce qui représente une source d'incertitudes supplémentaires. Dans certains cas, avec l'utilisation de sondes optiques, on peut éviter cet inconvénient. La combinaison de plusieurs pointes sensibles placées dans le même voisinage permet en principe de déterminer, pour chaque interface qui passe par toutes les pointes sensibles, la vitesse d'interface, $v_{i}$ et la direction, $\boldsymbol{n}$, du vecteur normal de l'interface. L'aire interfaciale locale moyennée s'obtient alors par l'identité,

$$
\gamma=\frac{l}{T} \sum_{j} \frac{1}{\left|\boldsymbol{v}_{i j} \cdot \boldsymbol{n}_{j}\right|},
$$

où $T$ représente le temps d'évaluation de la moyenne et la sommation porte sur toutes les interfaces passant au point $\boldsymbol{x}$ pendant le temps d'intégration $T$.

La mise en œuvre de ce principe est la plus simple en écoulement à bulles où avec seulement deux sondes, on peut déterminer un histogramme des temps de vol. La détermination de la vitesse des bulles, des diamètres moyens et de l'aire interfaciale locale nécessite une interprétation de cette donnée avec des hypothèses assez restrictives dont les plus fréquentes sont : l'écoulement a une direction privilégiée, les bulles sont sphériques et de même diamètre, les vitesses d'interface et les directions des normales sont décorrélées, etc. Hibiki et al. [10] ont mis au point une bisonde résistive dont chaque élément est constitué d'un fil de platine de $0,127 \mathrm{~mm}$ de diamètre et de longueur sensible $0,4 \mathrm{~mm}$. Les deux pointes sensibles sont espacées de $3 \mathrm{~mm}$ et sont alignées selon la direction privilégiée de l'écoulement (figure 4). Pour obtenir des valeurs statistiquement fiables dans des écoulements d'eau et d'air à bulles de quelques millimètres de diamètre à des vitesses de l'ordre de 1 à quelques $\mathrm{m} / \mathrm{s}$, il convient d'échantillonner le signal brut à plusieurs dizaines de $\mathrm{kHz}$ et d'avoir 2000 événements au moins pour obtenir un diamètre moyen de Sauter à $12 \%$ près selon ces auteurs.

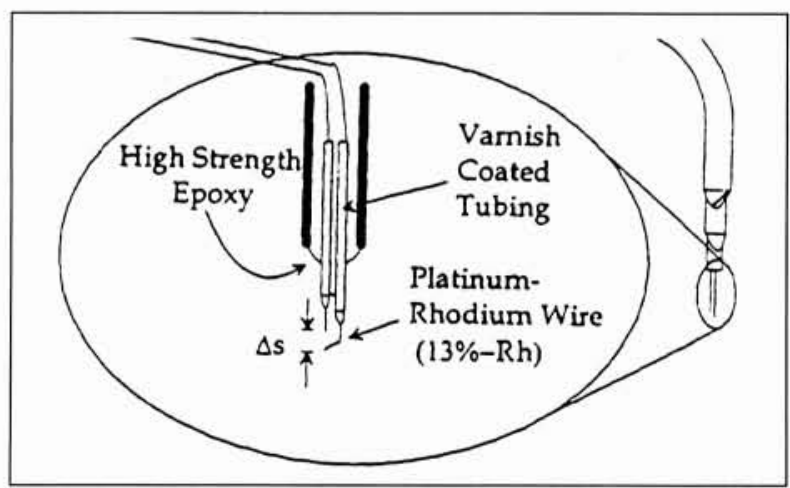

4. Schéma d'une double sonde résistive d'après [10]. 


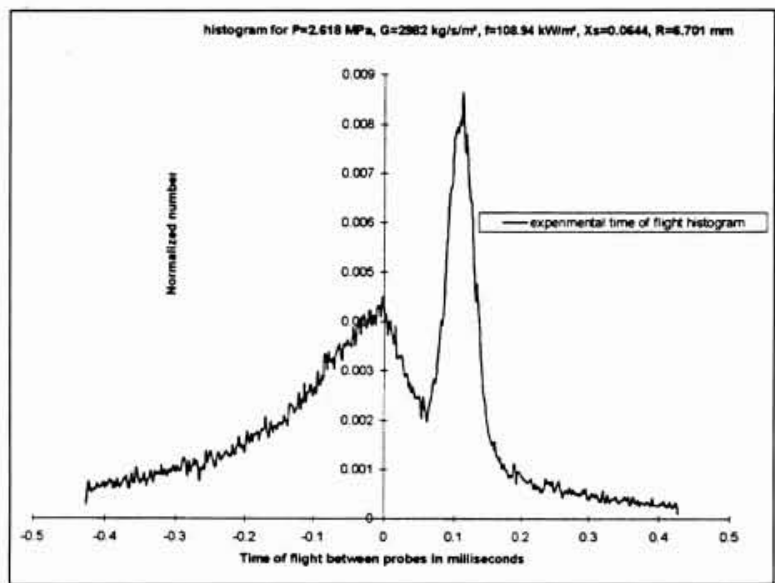

5. Exemple d'histogramme de temps de vol. Le pic centré sur zéro correspond aux fausses coüncidences tandis que le pic de droite correspond au temps de vol le plus probable d'après [11].

Cette méthode comporte de nombreuses sources d'incertitudes cachées, liées aux hypothèses de traitement et qu'il est impossible d'évaluer a priori. Garnier [11] a tenté avec succès d'utiliser des simulations numériques pour évaluer l'influence de ces hypothèses, notamment l'existence d'une distribution de taille, et la non uniformité des profils de vitesse et de diamètres moyens. De plus, pour des écoulements de fréon qui est un isolant électrique, Garnier a préféré utiliser des sondes optiques doubles pour étudier l'ébullition en convection forcée dans un tube. Dans ces expériences, les bulles peuvent être assez petites, notamment lorsque la pression augmente (jusqu'à $100 \mu \mathrm{m}$ ) et l'exploration complète du domaine de fonctionnement de l'installation DEBORA [11] montre qu'il faut s'attendre à trouver des bulles de $20 \mu \mathrm{m}$ à des vitesses de $7 \mathrm{~m} / \mathrm{s}$ environ.

Les contraintes technologiques sont ici beaucoup plus sévères que pour les écoulements décrits par Hibiki et al. [10] et des techniques originales ont été mises au point pour produire et qualifier des bisondes dont les pointes sont espacées de $400 \mu \mathrm{m}$ seulement et dont la partie sensible est plus petite que $20 \mu \mathrm{m}$. L'obtention des temps de vol les plus courts avec une précision relative de $1 \%$ nécessite d'échantillonner à $20 \mathrm{MHz}$. Le stockage de plusieurs minutes de signal étant technologiquement impossible, Garnier a montré qu'il était néanmoins possible d'analyser de façon satisfaisante les données en construisant en temps réel : les histogrammes de temps de vol, les histogrammes de temps de présence de la vapeur, la fréquence de passage des bulles sur la première fibre et les histogrammes de temps de montée et de descente des fronts sur la première fibre.

La figure 5 montre que lorsque la fréquence de passage des bulles augmente et est de l'ordre de $12 \mathrm{kHz}$, les fausses coïncidences sont nombreuses : quand une bulle touche la première fibre, une autre peut toucher la seconde et interférer. Grâce à l'histogramme des temps de vol, on peut substituer la valeur la plus probable à la valeur moyenne des temps de vol et réduire très sensiblement les incertitudes.

Garnier [11] montre de plus qu'on peut utiliser les histogrammes de temps de montée et de descente du signal pour déterminer les vitesses des bulles: Grâce à cette information on peut construire l'histogramme de taille de bulles dont on peut déduire l'aire interfaciale avec une seule fibre. La retombée la plus intéressante de la démarche est de pouvoir, avec ces informations, reconstituer par simulation les histogrammes de temps de vol et pouvoir vérifier ainsi la pertinence de l'hypothèse de sphéricité des bulles. Lorsque cette dernière est mise en défaut l'histogramme mesuré est moins large que l'histogramme simulé, ce que montre la figure 6.

En dehors de la technique photographique, il n'existe pas de technique de référence pour la mesure de l'aire interfaciale locale. La seule validation possible est la comparaison, par intégration de mesures locales, avec la détermination de l'aire interfaciale volumique moyenne. Cette dernière grandeur peut être déterminée en écoulement à bulles à partir de l'atténuation ultrasonore à des longueurs d'onde de l'ordre de la taille des bulles. Bensler [12] a mis en œuvre cette technique et a étudié l'influence systématique des effets de champ proche, de taille des capteurs, pour des écoulements d'eau et d'air dont les bulles avaient une taille moyenne de 2 à $6 \mathrm{~mm}$. Cette technique a depuis été mise en cuvre par Boyer [13], pour des écoulements eau air dont les bulles avaient une taille moyenne de l'ordre de $0,5 \mathrm{~mm}$. L'atténuation d'un faisceau d'ultrasons est proportionnelle au nombre
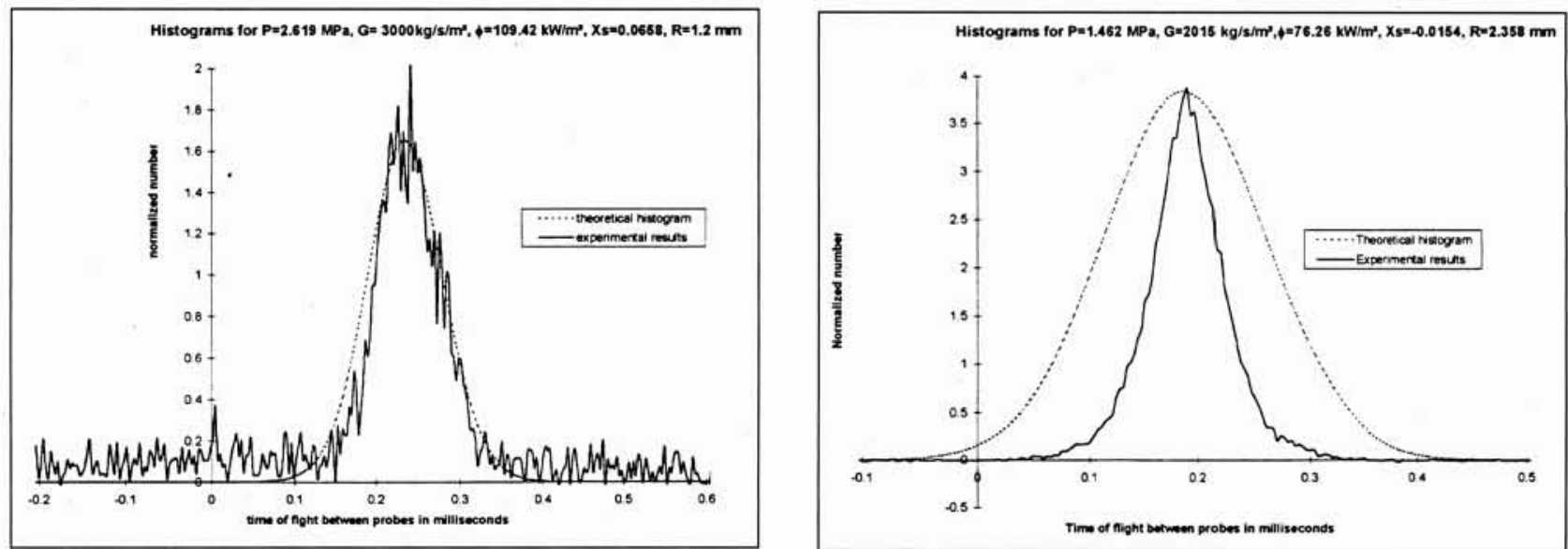

6. Comparaison entre un histogramme expérimental de temps de vol et une simulation basée sur la granulométrie mesurée. A gauche, à la plus forte pression les bulles sont sphériques, à droite, à plus basse pression les bulles s'aplatissent et l'histogramme mesuré est plus resserré que l'histogramme reconstitué d'après Garnier [11]. 
de bulles interceptées et à leur surface projetée. De plus, la section efficace de diffusion dépend légèrement de la taille, ce qui par l'analyse de l'atténuation fréquentielle, permet de déterminer simultanément, le taux de présence moyen et l'aire interfaciale moyenne sans intrusion [12]. L'influence de la diffusion multiple qui intervient lorsque le nombre de bulles augmente a été étudiée et modélisée par Boyer [13].

Il n'y pas eu, à notre connaissance, de validation croisée des techniques utilisant des bisondes et de la méthode ultrasonore et on ne peut que l'encourager de nos vœux.

\section{IV — LA DÉTERMINATION DES VITESSES ET DE LEURS FLUCTUATIONS}

La détermination des vitesses locales pour une structure et une direction arbitraires d'un écoulement diphasique est encore, à ce jour, un défi non relevé. Des progrès importants ont néanmoins été enregistrés pour les écoulements à bulles où l'on peut déterminer par anémométrie thermique (film chaud) ou par anémométrie laser Doppler, la vitesse instantanée de la phase liquide. La mise en œuvre de l'anémométrie laser est tout de même limitée aux faibles taux de vide d'autant plus que l'épaisseur de milieu traversé est grande, tandis que l'anémométrie à film est adaptée à des écoulements possédant une direction privilégiée d'écoulement et un taux de turbulence modéré. Les quantités que l'on cherche à déterminer sont celles que l'on rencontre dans les modèles d'écoulements turbulents à bulles : vitesses moyennes et tenseur de Reynolds de la phase continue. Les expériences récentes ainsi que leur interprétation par des modèles d'écoulements turbulents comprenant éventuellement une équation de transport d'aire interfaciale sont décrits par Grossetête [18] et Morel [19].

Le problème principal réside dans l'interprétation du signal anémométrique et la discrimination des phases. En effet, pour un film fonctionnant à température constante, la mesure de la vitesse est déduite de l'échange de chaleur qui est en proportion d'une puissance fractionnaire $(\sim 0,5)$ de la vitesse. Lorsque la sonde perce une interface, son assèchement provoque une chute brutale du transfert de chaleur et lors du remouillage l'échange se rétablit rapidement en montrant un surcroît de vitesse systématique $[10,15,14]$. Ces caractéristiques sont bien visibles aux figures 7 et 8 .
La question centrale que pose le traitement de ces signaux est bien résumée par Suzanne : le pic de vitesse observé à la suite de la phase de remouillage fait-il partie ou non du signal de vitesse du liquide ? La comparaison avec un signal d'anémométrie laser présenté à la figure 8 obtenu pratiquement au même point montre la même tendance. Cependant la question n'est pas tranchée pour autant [15] sachant que la discrimination des phases est difficile en anémométrie laser car elle nécessite, en principe, l'utilisation d'une autre sonde locale. De la réponse à cette question dépend la validation des modèles de turbulence en écoulement à bulles.

Lorsque l'écoulement diphasique a une structure arbitraire, il n'est pas possible d'obtenir une information locale sur la vitesse. En écoulement monophasique laminaire, différentes techniques d'imagerie par résonance magnétique nucléaire (RMN) permettent d'obtenir des distributions de vitesse dans une conduite [16]. En écoulement monophasique turbulent ou diphasique l'équipe de J. Leblond a démontré que l'on pouvait déterminer par des séquences $\mathrm{RMN}$ appropriées, la distribution de vitesse liquide dans un volume de conduite. On peut également déterminer précisément le taux de présence moyen et la vitesse moyenne, ce qui permet une détermination absolue du débit de liquide en écoulement diphasique d'eau et d'air par exemple. A notre connaissance ceci est unique. Ainsi, Leblond et al. [17] ont pu déterminer simultanément de le débit de liquide et le taux de vide moyen d'un écoulement transitoire résultant de la vidange d'un réservoir (Figure 9). En mettant au point d'autres séquences RMN, Leblond et al. [17] pensent pouvoir déterminer les moyennes surfaciques de vitesses phasiques instantanées à une fréquence répétition pouvant atteindre le $\mathrm{kHz}$. Cette technique permet un premier accès aux échelles de fluctuations de vitesse liquide en écoulement diphasique ce qui est la première étape vers la caractérisation puis, la modélisation, de la turbulence ou de l'intermittence en écoulement diphasique non structuré.

Pour un écoulement permanent en moyenne, il est possible de marquer le fluide par des gradients de champ transversaux. D'un point de vue matériel cela consiste à ajouter une ou plusieurs bobines de gradient aux dispositifs existant à l'ESPCI. Il est donc possible de localiser l'information dans la section de la conduite et d'obtenir alors un profil de vitesse moyenne et de caractériser ces fluctuations de

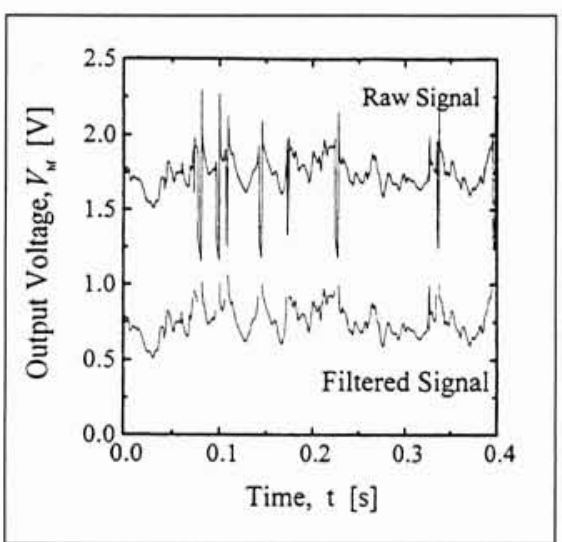

7. Signal d'anémométrie thermique en écoulement à bulles selon [10].

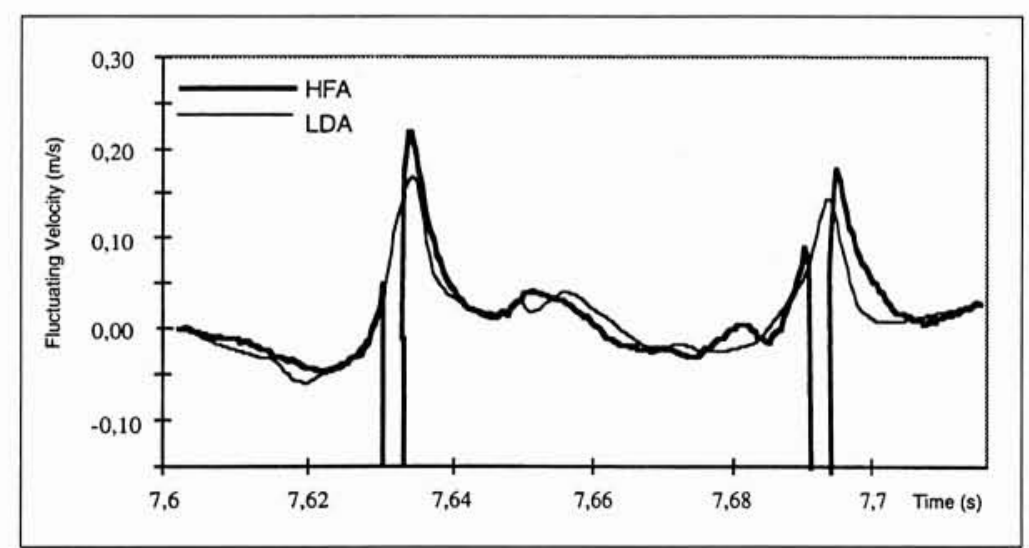

8. Signal d'anémométrie thermique non traité et signal laser filtré en écoulement à bulles selon [15]. 


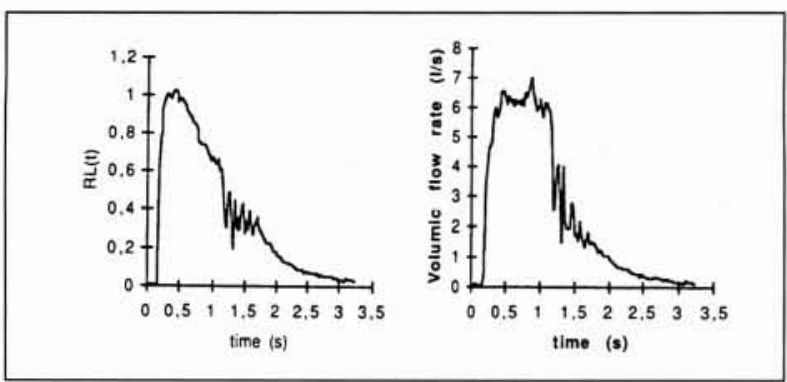

9. Débit et taux de vide instantanés dans la section pendant la vidange d'un réservoir d'eau selon Leblond et al. [17]

vitesse. Cette technique, bien que limitée à des géométries tubulaires, est cependant indépendante du régime d'écoulement et non intrusive ; il est donc absolument nécessaire de la développer en tant que technique de référence pour la mesure des vitesses en écoulement diphasique.

\section{CONCLUSIONS}

Les techniques de sondes locales multiples, de tomographie à rayons $\mathrm{X}$ et d'imagerie $\mathrm{RMN}$, permettent déjà et permettront moyennant des développements prévisibles de déterminer avec précision et un degré d'intrusion faible, le taux de vide local, l'aire interfaciale moyenne locale et les vitesses phasiques pour des régimes d'écoulement diphasique pouvant être arbitraires. Ces grandeurs interviennent au premier ordre dans les modèles d'écoulement diphasique tridimensionnels déstructurés que réclament les applications industrielles et on ne peut que recommander les développements nécessaires à l'avènement de leur maturité.

\section{Références}

[1] Williams, R. A. \& BEck M. S., 1995, Process tomography, principles, techniques and applications, Williams, R. A. \& Beck M. S. Eds., Butterworth Heinemann.

[2] OVAcik L., Lin \& Jones O. C., 1997, Progress in electrical impedance imaging of binary media. 2: experimental developments and results, Proc. OECD/CSNI Specialist Meeting on advanced instrumentation and measurement techniques, Santa Barbara, CA, March 17-20, 1997.

[3] OVACIK L., LIN, J. T. \& JoNES O. C., 1997, Progress in electrical impedance imaging of binary media. 1: analytical and numerical methods, Proc $O E C D / C S N I$ Specialist Meeting on advanced instrumentation and measurement techniques, Santa Barbara, CA, March 17-20, 1997.

[4] Lemonnier H. \& Peytraud J.-F., 1997, Is 2D impedance tomography a reliable technique for two-phase flows ? Proc. OECD/CSNI Specialist Meeting on advanced instrumentation and measurement techniques, Santa Barbara, CA, March 17-20, 1997.

[5] Mishima K. \& HibIKI T., 1997, Development of high frame rate neutron radiography and quantitative measurement method for multiphase flow research, Proc, OECD/CSNI Specialist Meeting on advanced instrumentation and measurement techniques, Santa Barbara, CA, March 17-20, 1997.

[6] BeCKer M. C., BONAZZA, R. \& Corradini L., 1997, Measurements of void fraction in water molten tin system by $\mathrm{X}$ ray absorption, Proc. $O E C D / C S N I$ Specialist Meeting on advanced instrumentation and measurement techniques, Santa Barbara, CA, March 17-20, 1997.

[7] ANgelinI S. \& Theofanous T. G., 1997, Void fraction measurements by means of flask X-ray radiography, Proc. OECD/CSNI Specialist Meeting on advanced instrumentation and measurement techniques, Santa Barbara, CA, March 17-20, 1997.

[8] Taneka, N., Asano, H., FujI T. \& Matsubayashi, M., 1997, Three dimensional void fraction measurement of steady two-phase flow by neutron radiography, Proc, OECD/CSNI Specialist Meeting on advanced instrumentation and measurement techniques, Santa Barbara, CA, March 17-20, 1997

[9] Hori K., Fujimoto T., KaWanishi K. \& Nishikawa H., 1997, Advanced high speed X-ray CT scanner for measurement and flow visualization of multiphase flow, Proc. OECD/CSNI Specialist Meeting on advanced instrumentation and measurement techniques, Santa Barbara, CA, March 17. 20, 1997.

[10] Hibiki T., Hogsetr S. \& IsHII M., 1997, Local measurement of interfacial area, interfacial velocity and liquid turbulence in two-phase flow, Proc. OECD/CSNI Specialist Meeting on advanced instrumentation and measurement techniques, Santa Barbara, CA, March 17-20, 1997.

[11] GARNIER J., 1997, Measurement of local two-phase flow pattern in boiling R12 simulating PWR conditions with multiple optical probes, Proc. $O E C D / C S N I$ Specialist Meeting on advanced instrumentation and measurement techniques, Santa Barbara, CA, March 17-20, 1997.

[12] BeNSLeR H., 1990, Détermination de l'aire interfaciale, du taux de vide et du diamètre moyen de Sauter dans un écoulement à bulles à partir de l'atténuation d'un faisceau d'ultrasons, Doctorat de l'Institut National Polytechnique de Grenoble.

[13] BOYER СH., 1996, Etude d'un procédé de mesure des débits d'un écoulement triphasique eau-huile-gaz, Doctorat de I'Institut National Polytechnique de Grenoble.

[14] Sharma S., Lewis S. \& Kojasoy G., 1997, Local studies in horizontal gas liquid slug flow, Proc. OECD/CSNI Specialist Meeting on advanced instrumentation and measurement techniques, Santa Barbara, CA, March 17-20, 1997

[15] Suzanne C., Euingsen K., Risso F. \& Roig V., Local measurements in turbulent bubbly flow, Proc. OECD/CSNI Specialist Meeting on advanced instrumentation and measurement techniques, Santa Barbara, CA. March 17-20, 1997

[16] Reyes J. N. JR. \& LaF A. Y., 1997, The use of magnetic resonance imaging for quantifying flow patterns and transitions, Proc. OECD/CSNI Specialist Meeting on advanced instrumentation and measurement techniques, Santa Barbara, CA, March 17-20, 1997.

[17] LeBlond J., JAVelot S., LeBrun D. \& LeBon L., 197, Two-phase flow characterization by nuclear magnetic resonance, Proc. OECD/CSNI Specialist Meeting on advanced instrumentation and measurement techniques, Santa Barbara, CA, March 17-20, 1997.

[18] Grossetete, C., 1995, Caractérisation expérimentale et simulations numériques de l'évolution d'un écoulement diphasique à bulles ascendant dans une conduite verticale. Doctorat de l'Ecole Centrale Paris.

[19] MORFL C., 1997, Modélisation multidimensionnelle des écoulemengts diphasiques gaz liquide. Application à la simulation des écoulements a bulles ascendants en conduite verticale. Doctorat de l'Ecole Centrale Paris. 